\title{
Effects of Numerical Diffusion on the Computation of Viscous Supersonic Flow Over a Flat Plate
}

\author{
Paragmoni Kalita $^{1}$ (D) Anoop Kumar Dass ${ }^{2} \cdot$ \\ Abhishek Sarma ${ }^{1}$
}

Published online: 16 September 2015

(C) Springer India Pvt. Ltd. 2015

\begin{abstract}
The effects of numerical diffusion on the computation of supersonic viscous flow over a flat plate at zero incidences are numerically investigated. The inviscid flux terms in the Navier-Stokes equations are computed using three schemes, namely, van Leer's Flux Vector Splitting, Liou and Steffen's Advection Upstream Splitting Method (AUSM) and Jaisankar and Raghurama Rao's Diffusion Regulated Local Lax Friedrichs (DRLLF) schemes. The results are correlated with the inherent numerical diffusion of these schemes. The study is also motivated by the necessity to examine whether reduced artificial viscosity can be used with the DRLLF scheme in computing viscous flows than was suggested in the original paper on inviscid computation. It is demonstrated that reduced artificial viscosity is not only possible, but it results in a scheme that is very efficient in the computation of the standard supersonic viscous flow over a flat plate, in that it is comparable in accuracy to the AUSM scheme in the boundary layer and better than it in shock resolution. Even with the reduced artificial viscosity suggested in this paper the DRLLF scheme shows good convergence behaviour comparable with the other two schemes.
\end{abstract}

Keywords Inviscid flux · Viscous flux · Gradient · Numerical diffusion · Entropy

\section{Introduction}

This work is concerned with the computation of viscous supersonic flow of air over a flat plate at zero incidence [30] on a finite volume framework. The governing equations for the fluid flow are the Navier-Stokes Equations. When applied to high-speed flows, these equations exhibit hyperbolic-elliptic behavior. These equations are numerically solved for flow at high Mach numbers.

Paragmoni Kalita

paragmk@tezu.ernet.in

1 Department of Mechanical Engineering, Tezpur University, Tezpur, Assam 784028, India

2 Department of Mechanical Engineering, Indian Institute of Technology, Guwahati, India 


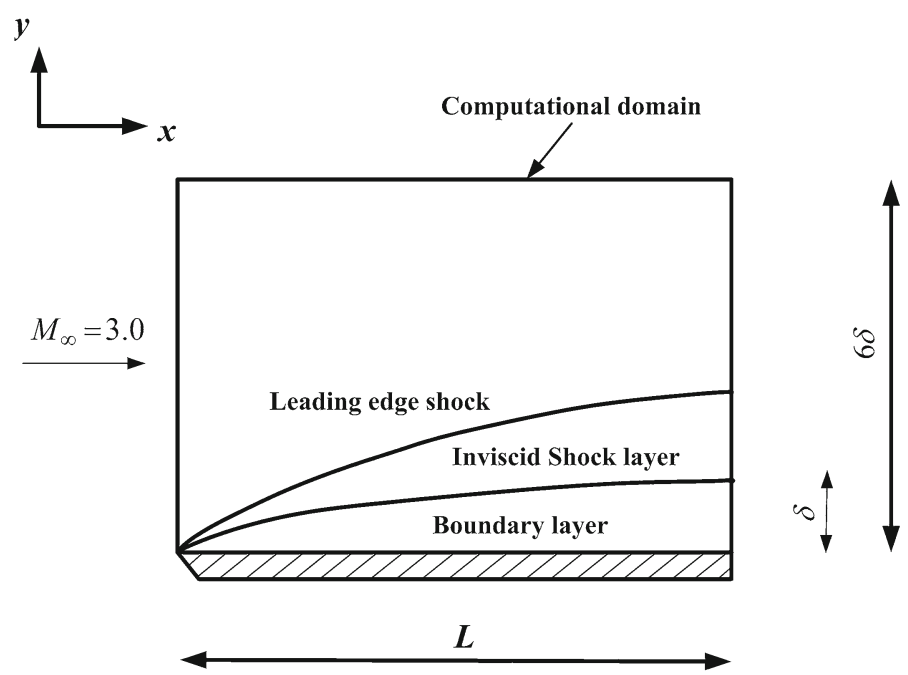

Fig. 1 The various zones for viscous supersonic flow over a flat plate

For viscous flows, because of the no-slip condition on the wall, fluid at the wall and some neighbourhood has to be necessarily subsonic. In case of a supersonic freestream, this can happen only through the shock emanating from the leading edge of the flat plate. The region between the surface and the shock is the shock layer. Also owing to the viscous effects, a boundary layer develops on the surface. In general, the shock layer can be characterized by the region of viscous flow and inviscid flow as shown in Fig. 1. It may be noted, that dissipation of kinetic energy within the boundary layer can cause high temperature gradients near the solid surface. This may lead to high heat transfer rates.

Aircrafts flying through the atmosphere at supersonic speeds have slender shapes with very low wall curvature resembling the flow over a flat plate. Eckert [12] suggested that the solution of supersonic viscous flow over a flat plate could be extended to obtain parameters like skin friction coefficient, recovery factor, and convective heat transfer coefficient for high-speed flow over slender surfaces maintained at constant temperatures. This reaffirms the importance of the classical problem of the boundary layer over a flat plate.

Various studies have been done on the computation of high-speed viscous flows over flat plates. Aliabadi et al. [2] presented a finite element-based computation of viscous compressible flows based upon the conservation and entropy variables formulations. Savelyev [28] showed a numerical simulation of laminar viscous supersonic flow over a flat plate with a rectangular cavity. Drikakis and Durst [10] presented a numerical assessment of the effect of angle of incidence and Reynolds number on the flow separation for viscous supersonic flow past a flat plate. Aoki et al. [4] carried out numerical analysis of supersonic rarefied gas flow past a flat plate. Deshpande et al. [9] carried out a computational study of viscous supersonic flow over a flat plate with moving protrusion. Abbas and Nordström [1] demonstrated that in the numerical computation of the Navier-Stokes equations involving boundary layers it is advantageous to use weak no-slip boundary conditions. Arnal and Vermeerch [5] gave an overview of the compressibility effects, free-stream Mach number and wall temperature effects, on boundary layer laminar-turbulent transition from incompressible flow to low supersonic Mach number. Iyer et al. [14] investigated the boundary layer transition in high-speed flows due to roughness. Fang et al. [13] presented an optimized low-dissipation 
monotonicity-preserving scheme for numerical simulations of high-speed turbulent flows. $\mathrm{Xu}$ and Weng [32] proposed a high order accurate and low dissipation method for unsteady compressible viscous flow computation on helicopter rotor in forward flight. However, a comprehensive study of the effect of numerical diffusion on the computation of viscous supersonic flows over a flat plate under isothermal and adiabatic wall conditions is not available in the literature.

The inviscid flux terms across the cell interfaces in the Navier-Stokes equations for highspeed flows can be numerically computed by either a central or an upwind scheme. van Leer's FVS [3], Liou and Steffen's AUSM [24], Steger and Warming's FVS [29], Roe's Flux Difference Splitting [27] etc. are some of the popular upwind methods. McCormack's scheme [25], Local Lax-Friedrichs (LLF) or Rusanov's scheme [23], Jameson et al.'s JST scheme [17] etc. are examples of central schemes.

For numerical stability, a minimum level of numerical diffusion or artificial viscosity is to be added to the central discretization of the flux terms [11,17]. Although the upwind schemes are the natural choices for high-speed flow computations, the central schemes also bear some advantages like algorithmic simplicity and easier manoeuvrability of the numerical diffusion considering both accuracy and stability. Entropy generation along the flow may be considered as a parameter to quantify the relative numerical diffusion of various schemes [11,26].

Jaisankar and Raghurama Rao [15] introduced a Diffusion Regulation (DR) parameter which was used to regulate the inherent numerical diffusion of different schemes. Later on Jaisankar and Sheshadri [16] introduced another parameter called Directional Diffusion Regulation (DDR) parameter for introducing multidimensional physics into the computations. The DR parameter and DDR parameter were coupled with the LLF scheme and the new schemes were named as DRLLF [15] and DDRLLF [16] respectively. For the computation of the Euler equations for high-speed inviscid flows, the DRLLF scheme is found to be more competitive as compared to the DDRLLF scheme in terms of versatility, robustness, computational time and accuracy [19].

Kannan and Wang [22] used the DR parameter to improve the high order spectral volume formulation and demonstrated the potential of the diffusion regulation for viscous subsonic test cases. The spectral volume method is a recent high order method [20,21] for hyperbolic and elliptic conservation laws on unstructured grids. The performance of the DRLLF scheme for computing viscous supersonic flows, however, is an unexplored area. The van Leer's FVS scheme is known to be robust, although more diffusive especially for viscous flow [24]. The AUSM scheme was designed with less numerical diffusion to make it suitable for viscous flow computations as well. In the present work, a comparison of the solutions of the problem using the DRLLF, van Leer's FVS and AUSM schemes is done. Based upon the comparison, an analysis of the effect of numerical diffusion on the flow-features captured by the different schemes is presented. Emphasis on further improvement of the flow-field resolution by the DRLLF scheme is done because of its algorithmic simplicity compared with the other two schemes.

In the computation of viscous flows, physical diffusion is always present. Motivation is drawn from this fact to investigate the possibility of reducing the numerical diffusion of the DRLLF scheme for the computation of high-speed viscous flows than the level reported in the original paper for inviscid computations. Many methods exist for the computation of the gradients in the viscous flux terms [18]. Here these terms are computed by simple central discretization and using the Green's theorem [6]. Both these methods are combined with the three inviscid flux schemes and the results are compared. The equivalence of the two methods for computing the gradients across the cell interfaces in a uniform Cartesian grid is verified. 
This paper is organized in five sections. "Governing Equations" section presents the Navier-Stokes equations of gas dynamics. The DRLLF scheme is presented in "The Schemes for Computing the Inviscid and Viscous Fluxes" section along-with brief introductions of the van Leer's FVS scheme, the AUSM scheme and the Green's method for gradient computations. "Results and Discussion" section presents the results and discussion on the various computations. The steady state velocity, temperature and entropy profiles at the trailing edge of the plate for adiabatic and isothermal wall conditions are plotted for all the combinations of the schemes considered in this work. An analysis of the relative numerical diffusion for the different schemes is carried out by studying the velocity, temperature and entropy profiles at the trailing edge. "Summary and Conclusions" section summarizes the results along with concluding remarks.

\section{Governing Equations}

The Navier-Stokes equations for 2D compressible flow can be written as [8],

$$
\frac{\partial \mathbf{U}}{\partial t}+\frac{\partial \mathbf{F}_{I}}{\partial x}+\frac{\partial \mathbf{G}_{I}}{\partial y}=\frac{\partial \mathbf{F}_{v}}{\partial x}+\frac{\partial \mathbf{G}_{v}}{\partial y}
$$

where $\mathbf{U}, \mathbf{F}$ and $\mathbf{G}$ denote the conserved variable vector, flux vector in the $x$-direction and flux vector in the $y$-direction respectively. Suffixes $I$ and $v$ denote inviscid and viscous fluxes respectively. The expressions for these vectors with usual notations are as follows.

$$
\begin{gathered}
\mathbf{U}=\left[\begin{array}{l}
\rho \\
\rho u \\
\rho v \\
\rho e_{m}
\end{array}\right], \quad \mathbf{F}_{I}=\left[\begin{array}{l}
\rho u \\
p+\rho u^{2} \\
\rho u v \\
\left(p+\rho e_{m}\right) u
\end{array}\right], \quad \mathbf{G}_{I}=\left[\begin{array}{l}
\rho v \\
\rho v u \\
p+\rho v^{2} \\
\left(p+\rho e_{m}\right) v
\end{array}\right] \\
\mathbf{F}_{v}=\left[\begin{array}{l}
0 \\
\tau_{x x} \\
\tau_{x y} \\
u \tau_{x x}+v \tau_{x y}-q_{x}
\end{array}\right], \quad \mathbf{G}_{v}=\left[\begin{array}{l}
0 \\
\tau_{x y} \\
\tau_{y y} \\
u \tau_{x y}+v \tau_{y y}-q_{y}
\end{array}\right]
\end{gathered}
$$

$q_{x}=-k \frac{\partial T}{\partial x}, q_{y}=-k \frac{\partial T}{\partial y}$ are the heat fluxes along the $x$ - and $y$-directions respectively.

The co-efficient of dynamic viscosity is taken as a function of temperature obeying the Sutherland's Law of viscosity given by [30],

$$
\frac{\mu}{\mu_{0}}=\left(\frac{T}{T_{0}}\right)^{\frac{3}{2}}\left(\frac{T_{0}+120}{T+120}\right)
$$

where, $\mu_{0}$ and $T_{0}$ are the reference viscosity and reference temperature respectively.

\section{The Schemes for Computing the Inviscid and Viscous Fluxes}

In the present work the van Leer's FVS, AUSM and DRLLF schemes are used to compute the inviscid flux terms in Eq. (1). To study the effects of the relative numerical diffusion of these schemes, only the first order accurate methods are used so that the effects of limiters used for higher order computations don't interfere with the analysis. The van Leer's FVS scheme splits the flux vector into two parts based upon the sign of the eigenvalues of the Flux Jacobian Matrix. The AUSM scheme splits the flux into convective and acoustic parts. The 
details of these two schemes are available in the original papers by Anderson et al. [3] and Liou and Steffen [24]. The DRLLF scheme is briefly discussed here since, in the later part of this paper, the formulation of the scheme is used to further reduce its numerical diffusion.

In the DRLLF scheme, the flux at the cell interface is given by [15],

$$
\mathbf{Q}_{\perp}=\frac{1}{2}\left(\mathbf{Q}_{\perp \mathrm{L}}+\mathbf{Q}_{\perp \mathrm{R}}\right)-\frac{1}{2} \Phi|\lambda|_{\max }\left(\mathbf{U}_{\mathrm{R}}-\mathbf{U}_{\mathrm{L}}\right)
$$

Here, $\mathbf{Q}_{\perp}$ is the flux normal to the cell face, subscripts $\mathrm{L}$ and $\mathrm{R}$ represent the cells on the upstream and downstream side of the cell interface, $|\lambda|_{\max }$ is the maxima of the local eigenvalues of the flux Jacobian matrices on either sides of the cell interface and $\Phi$ is the DR parameter that works based upon the jump in the normal Mach number across the cell interface as,

$$
\Phi= \begin{cases}\frac{\left(\Delta M^{2}+\delta^{2}\right)}{2 \delta}, & \text { for } \quad|\Delta M| \leq \delta, \\ |\Delta M|, & \text { for } \delta<|\Delta M| \leq 1 \\ 1.0, & \text { for } \quad|\Delta M|>1\end{cases}
$$

where $\Delta M$ is the jump in the normal Mach number across the cell interface. In the original paper [15] the value of $\delta=0.5$ was found to work for the inviscid test cases.

From Eq. (1) it is observed that the viscous flux terms contain the gradients of the flow variables. For a uniform Cartesian grid, it is a straightforward task of central differencing. However for non-orthogonal grids using this method for the gradient computation is not a straightforward task. For such grids, the Green's theorem can be used to compute the gradients at the cell interfaces with reasonable accuracy.

\section{Results and Discussion}

The viscous flow of air at Mach number 3.0 over a flat plate of length $L=2.85 \times 10^{-5} \mathrm{~m}$ is computed. The freestream pressure and temperature are taken as $101,325.0 \mathrm{~N} / \mathrm{m}^{2}$ and 288.16 $\mathrm{K}$ respectively. This corresponds to a Reynolds number $\left(\operatorname{Re}_{L}\right)$ of around 2000 at sea-level conditions. The Prandtl number is taken as 0.71 . Thus, the boundary layer developed on the plate can be considered laminar. The grid is formed using the following stretching function [7].

$$
\begin{aligned}
x & =\xi \\
\frac{y}{h} & =\frac{(\beta+1)-(\beta-1) B^{1-\eta}}{1+B^{1-\eta}}
\end{aligned}
$$

where $h$ is the height of the computational domain, $\xi, \eta$ are the transformed co-ordinates and $B=\frac{\beta+1}{\beta-1}, \beta(1<\beta<\infty)$ is the stretching parameter. As $\beta$ gets closer to 1 , more grid points are clustered near the wall. In the present work $\beta$ is taken as 1.1. Figure 2 shows a typical $40 \times 40$ grid obtained using Eq. (5) along-with the boundary conditions used in the computational domain.

The first order Euler-explicit time-integration method is used with a CFL number of 0.2 for all the computations. Both isothermal and adiabatic wall conditions are considered. For the isothermal wall condition, the wall is maintained at the freestream temperature. The $x$ direction is along the plate and the $y$-direction along the normal to the plate. The height of the computational domain normal to the plate is taken as six times the boundary-layer thickness at the trailing edge, which can be computed as follows [30]:

$$
\delta=\frac{5 L}{\sqrt{\operatorname{Re}_{L}}}=3.186 \times 10^{-6} \mathrm{~m}
$$




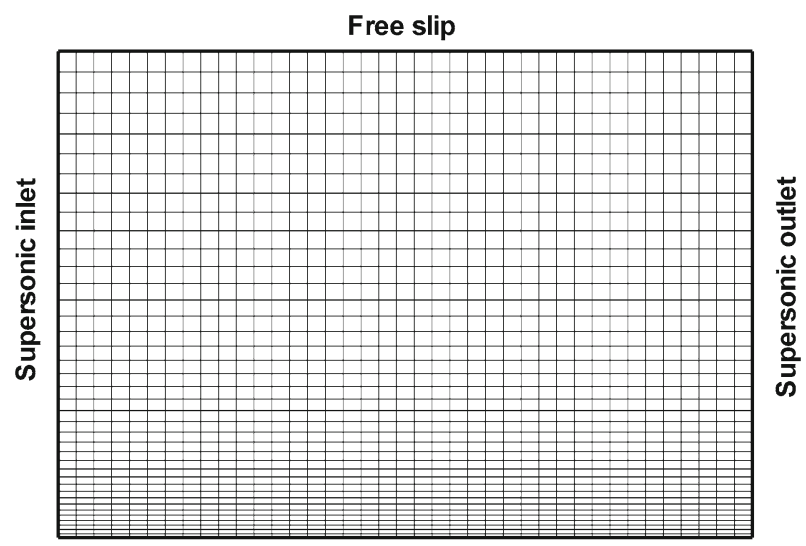

No slip adiabatic/isothermal wall

Fig. 2 A typical $40 \times 40$ grid showing the computational domain and the boundary conditions

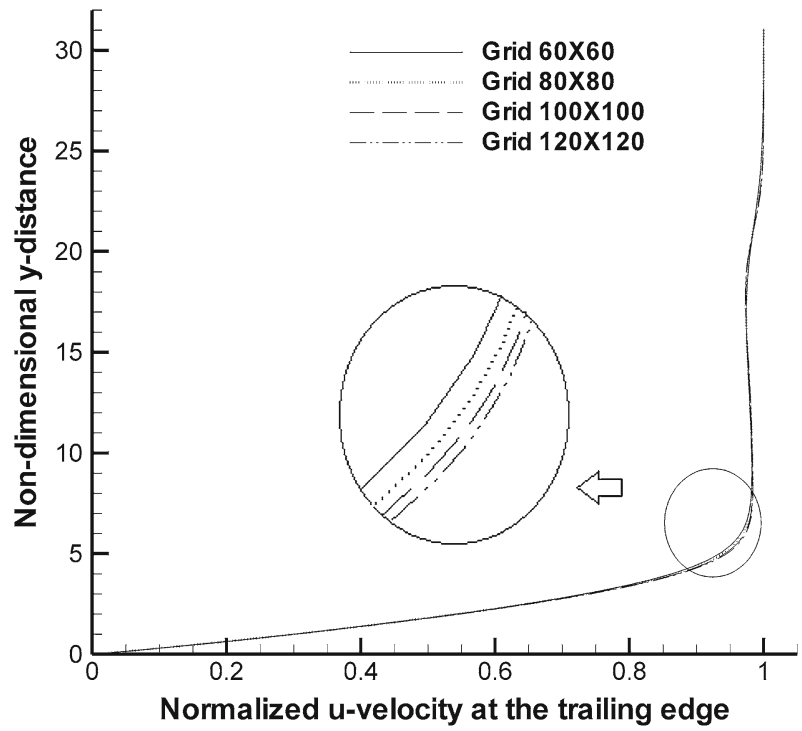

Fig. 3 Mesh-independence study showing the normalized $u$-velocity profile at the trailing edge of the flat plate under isothermal condition computed by the AUSM scheme

In the computation of the Navier-Stokes equations, since physical viscosity is already present, it is preferable to use a scheme with small numerical diffusion. From Eq. (4) it can be observed that a reduction in the value of $\delta$ implies reduced numerical diffusion in the DRLLF scheme. Hence in the present work, the DRLLF scheme is adopted by gradually decreasing $\delta$ from 0.5 to 0.1 in steps of 0.1 .

\section{Gradient Computation at Cell-Interfaces Using Central-Differencing}

A typical mesh-independence study is presented in Fig. 3 which shows the steady state normalized $u$-velocity profile at the trailing edge of the flat plate vs the non-dimensionalized 


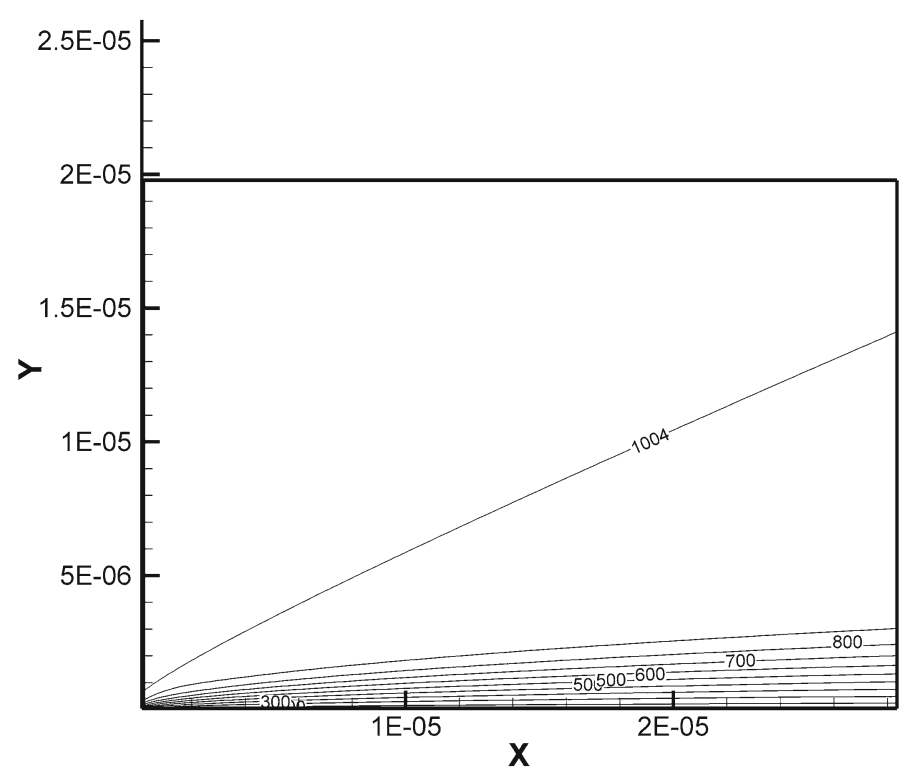

Fig. $4 u$-Velocity contour plot for the isothermal wall condition computed by the DRLLF scheme $(\delta=0.5)$

$y$-distance under isothermal condition computed using the AUSM scheme for the $60 \times$ $60,80 \times 80,120 \times 120$ and $140 \times 140$ grids. It is found that the $100 \times 100$ grid produces the mesh-independent results. All the subsequent results are presented for this grid. As suggested by Van Driest [31] the non-dimensionalized $y$-distance is given by

$$
\bar{y}=\frac{y}{x} \sqrt{\operatorname{Re}_{x}}
$$

where $\operatorname{Re}_{x}=\frac{\rho_{\infty} U_{\infty} x}{\mu_{\infty}}$ and $U_{\infty}$ is the freestream velocity.

The normalized u-velocity is obtained by

$$
\bar{u}=\frac{u}{U_{\infty}}
$$

The $u$-velocity contour plots computed using the DRLLF scheme with $\delta=0.5$ for the isothermal and adiabatic wall conditions are shown in Figs. 4 and 5 respectively. The leading edge shock and the boundary layer captured by the scheme can be seen clearly in the figures.

Figures 6 and 7 show the steady-state normalized $u$-velocity profile at the trailing edge of the plate vs. the non-dimensionalized $y$-distance for the isothermal wall and adiabatic wall conditions respectively. For clarity of the figures, the results of the DRLLF scheme for $\delta=0.1,0.2$ and 0.3 only are shown along-with those of AUSM and van Leer's FVS schemes. It is seen that the DRLLF scheme with $\delta=0.1$ and $\delta=0.2$ are in very close agreement with the AUSM scheme. This shows that by using a value of $\delta$ much lower than what was reported in the original paper for inviscid computations, it is possible to bring down the numerical diffusion of the DRLLF scheme and hence improve accuracy without causing numerical instability. The van Leer's FVS scheme predicts a much higher hydrodynamic boundary layer thickness, depicting the highly diffusive nature of the scheme.

Figures 8 and 9 show the steady-state normalized temperature profiles at the trailing edge for the isothermal and adiabatic wall conditions respectively. The normalized temperature is obtained by 


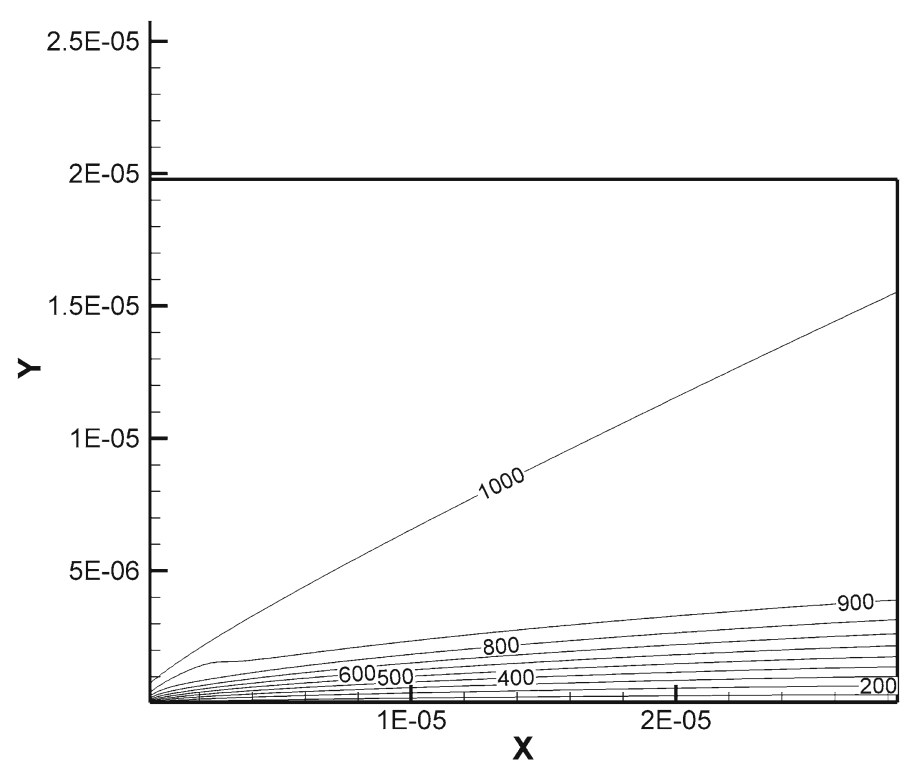

Fig. $5 u$-Velocity contour plot for the adiabatic wall condition computed by the DRLLF scheme $(\delta=0.5)$

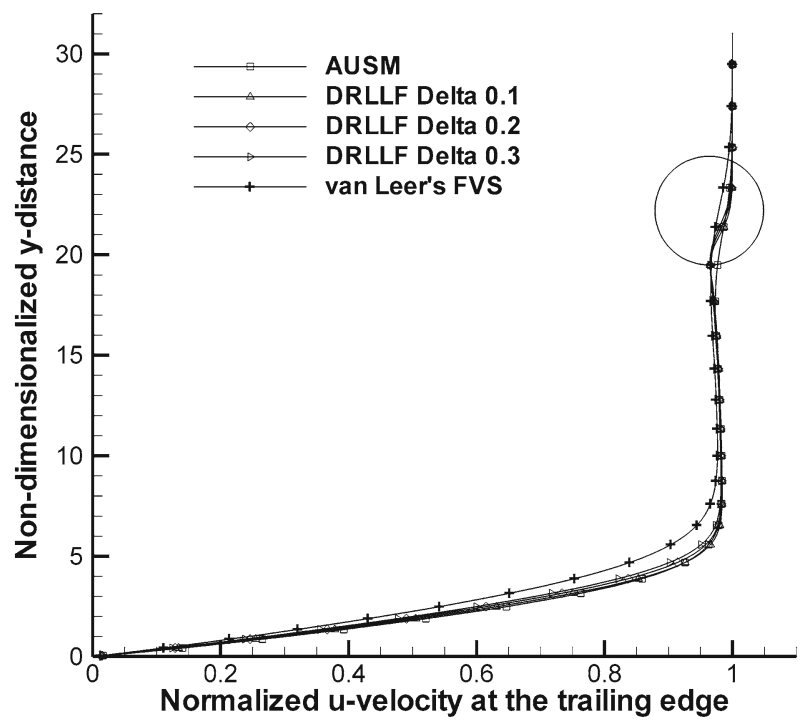

Fig. 6 Trailing edge $u$-velocity profile for the isothermal wall condition. Gradients at the cell-interfaces are computed by central-differencing

$$
\bar{T}=\frac{T}{T_{\infty}}
$$

Comparing Figs. 8 and 9, two interesting differences in the temperature profiles for the isothermal and adiabatic wall conditions can be noticed, which are correlated below with the numerical diffusion of the different inviscid flux schemes. 


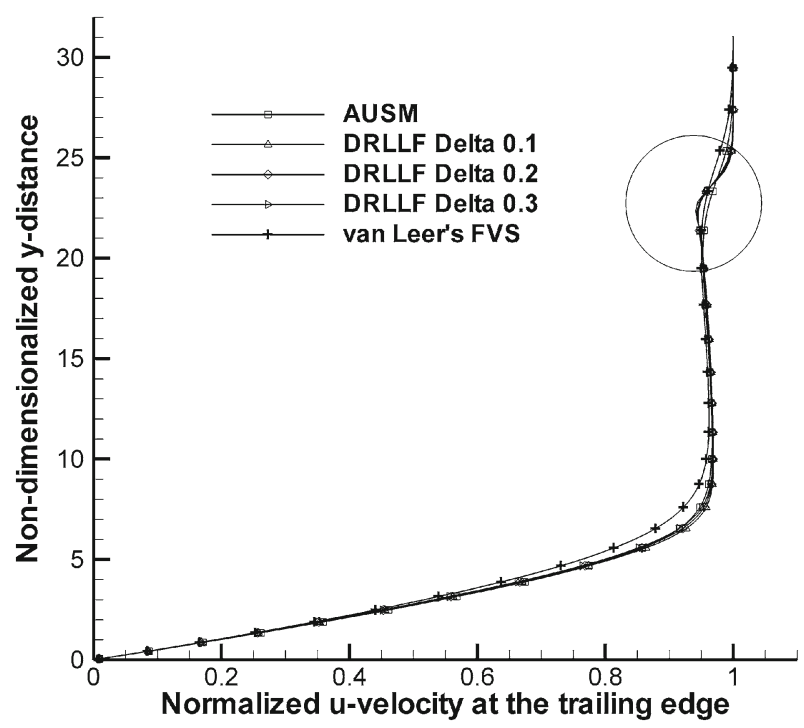

Fig. 7 Trailing edge $u$-velocity profile for the adiabatic wall condition. Gradients at the cell-interfaces are computed by central-differencing

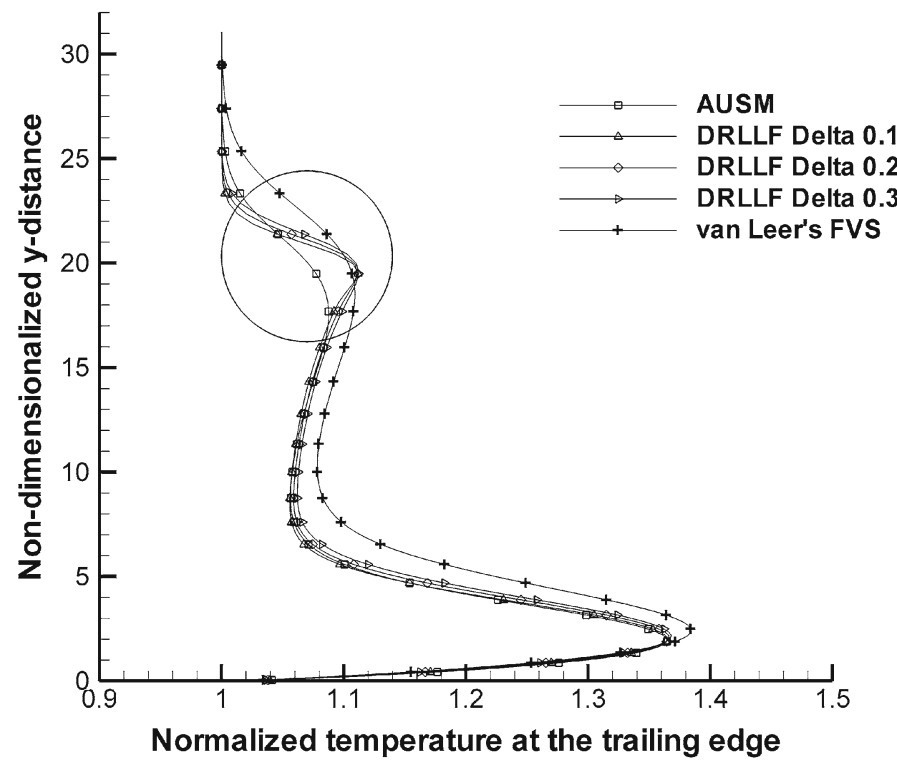

Fig. 8 Trailing edge temperature profile for the isothermal wall condition. Gradients at the cell-interfaces are computed using central-differencing

Figure 8 shows that for the isothermal wall condition, a point of maximum temperature is reached at some distance above the wall. It is seen that as the numerical diffusion increases the point of maximum temperature shifts upwards. Thus for the AUSM scheme this point is nearest to the wall, it moves up for the DRLLF scheme with increasing values of $\delta$ and is farthest from the wall for the van Leer's FVS. It is known that the ratio of the thicknesses of the 


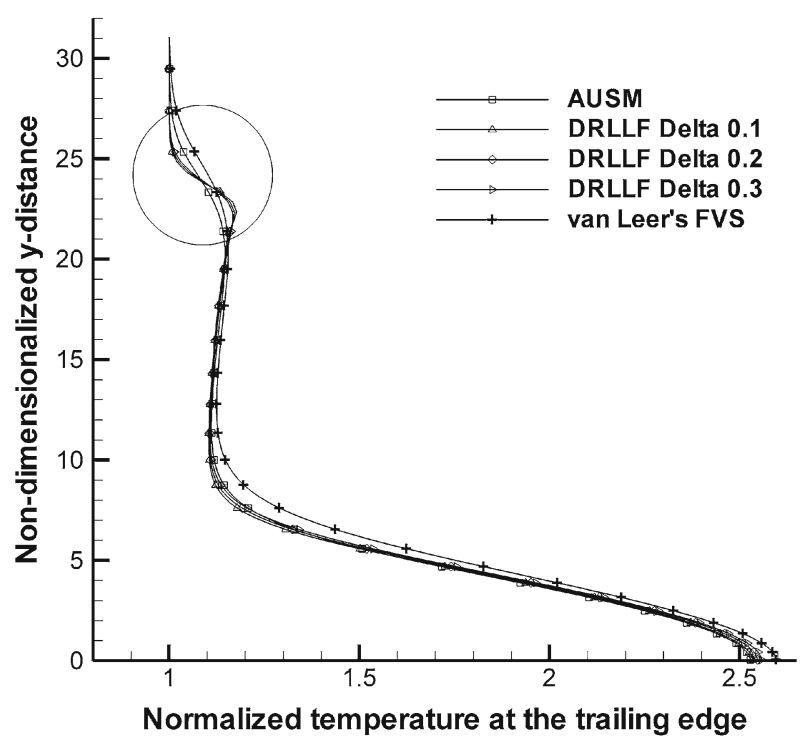

Fig. 9 Trailing edge temperature profile for the adiabatic wall condition. Gradients at the cell-interfaces are computed using central-differencing

hydrodynamic and thermal boundary layers is a function of the Prandtl number. Accordingly with increasing numerical diffusion, both the hydrodynamic as well as the thermal boundary thicknesses increase. When the thermal boundary layer thickness is high, the temperature gradients normal to the wall are less and vice-versa. This explains the reason for the upward shifting of the point of the maximum temperature with increasing numerical diffusion. The second observation is that the temperature variation trends above and below the point of the maximum temperature reverse for the isothermal wall condition. Below the point of maximum temperature, at a certain distance from the wall, the highest diffusive scheme shows the lowest temperature and vice-versa. This can be ascribed to the fact that for a scheme with lower diffusion the rate of increase of temperature to the maximum point is sharper. On the other hand, above the point of maximum temperature, at a certain $y$-location, the more diffusive scheme shows higher temperature. This can be attributed to the gradual rate of decrease of temperature from the maximum point for a scheme with higher diffusion.

Figure 9 shows that for the adiabatic wall condition, the maximum temperature point is located on the wall itself. This is thermodynamically justifiable since the high velocity of the incoming freestream is decelerated by the viscous effects on the wall, thereby converting large part of the kinetic energy into enthalpy. If we consider a streamline connecting the freestream and the first cell-centre above the wall, the summation of the kinetic energy and the static enthalpy at the grid point is equal to the freestream stagnation enthalpy. Since the freestream stagnation enthalpy is constant, hence if the fluid velocity shown by a scheme at the grid point is less, the static enthalpy obtained is more and vice-versa. For the highest diffusive scheme, because the fluid velocity at the cell-centroid just above the wall is the least so the static enthalpy is the maximum. Since temperature is proportional to the static enthalpy, hence the temperature shown at that point is also the maximum for the most diffusive scheme. For adiabatic wall, the temperature gradient at the wall is zero. So the temperature at the wall is also equal to the temperature at the cell-centroid just above the wall. The second observation 


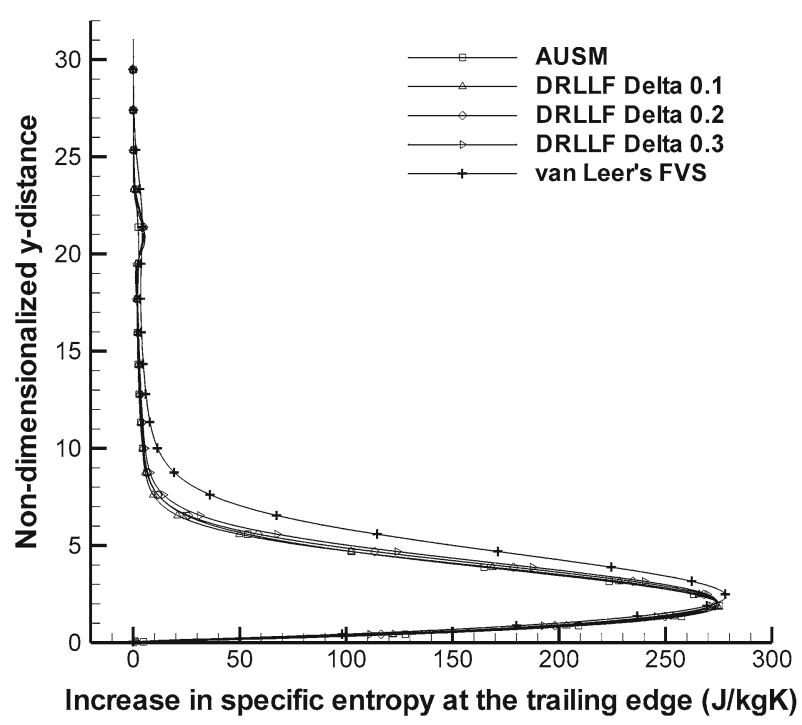

Fig. 10 Trailing edge specific entropy profile for the isothermal wall condition. Gradients at the cell-interfaces using computed by central-differencing

from Fig. 9 is that trend of the temperature variation for the adiabatic wall condition remains the same throughout the thermal boundary layer, i.e. the more diffusive scheme predicts a higher temperature. The reason for this occurrence is the lower temperature gradient within the thermal boundary layer for the more diffusive scheme. It can be noted that the van Leer's FVS scheme predicts erroneously high adiabatic wall temperature. However, the DRLLF scheme with $\delta=0.1$ and 0.2 agrees well with the AUSM scheme.

From Figs. 6, 7, 8 and 9 it can be further observed that the DRLLF scheme captures the leading edge shock better than the AUSM and van Leer's FVS schemes as shown by the encircled portions in these figures. The better performance of the DRLLF scheme in capturing weak shocks for inviscid flows was demonstrated earlier [19]. Even for the present viscous flow problem, since the shock is weak, the DRLLF scheme is seen to resolve the shock better than the other two schemes.

An attempt has been made to relate the change of entropy with the change of temperature. However, the numerical diffusion may not be the only factor influencing the entropy profile since there may be other effects such as heat transfer across the wall. Still it will not be out of place to study the change of entropy for the different schemes as it will provide an insight into the entropy generation due to diffusion. The free-stream conditions are considered as the datum and increase in entropy at any point over this datum is computed by using the thermodynamic identity,

$$
\Delta s=c_{p} \ln \left(\frac{T}{T_{\infty}}\right)-R \ln \left(\frac{p}{p_{\infty}}\right)
$$

where $c_{p}$ is the specific heat at constant pressure, $R$ is the characteristic gas constant, $T$ is the absolute temperature and $p$ is the pressure and subscript $\infty$ represents the freestream conditions. For compressible laminar boundary layer over a flat plate, the pressure ratios are small compared with the temperature gradients. Thus, the effect of the pressure term in Eq. (10) is not prominent as compared to that of the temperature term. As a result, the 


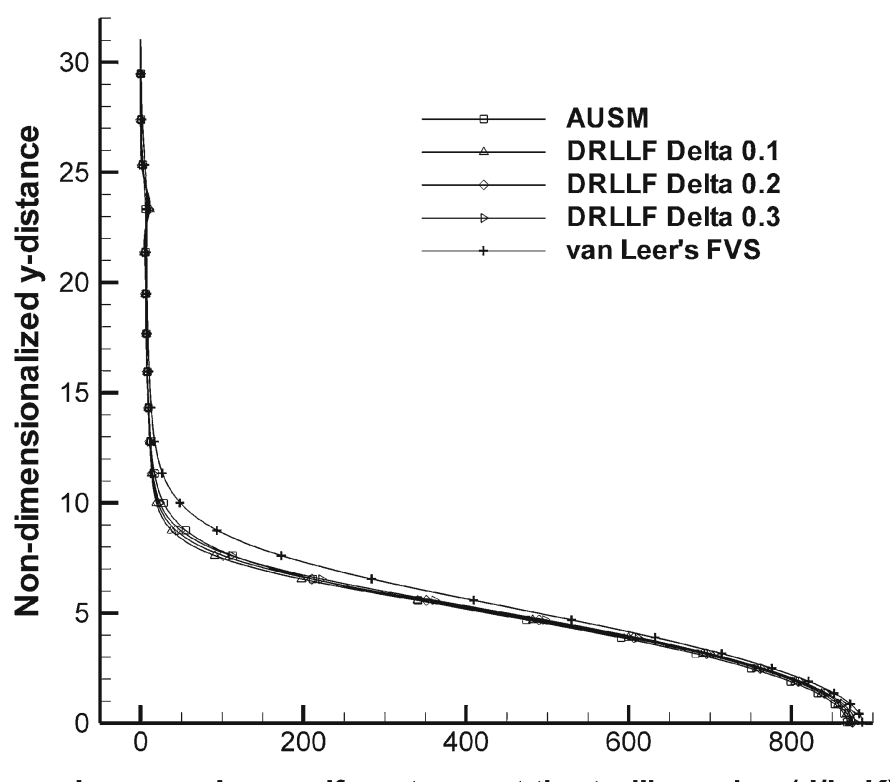

\section{Increase in specifc entropy at the trailing edge (J/kgK)}

Fig. 11 Trailing edge specific entropy profile for the adiabatic wall condition. Gradients at the cell-interfaces using computed by central-differencing

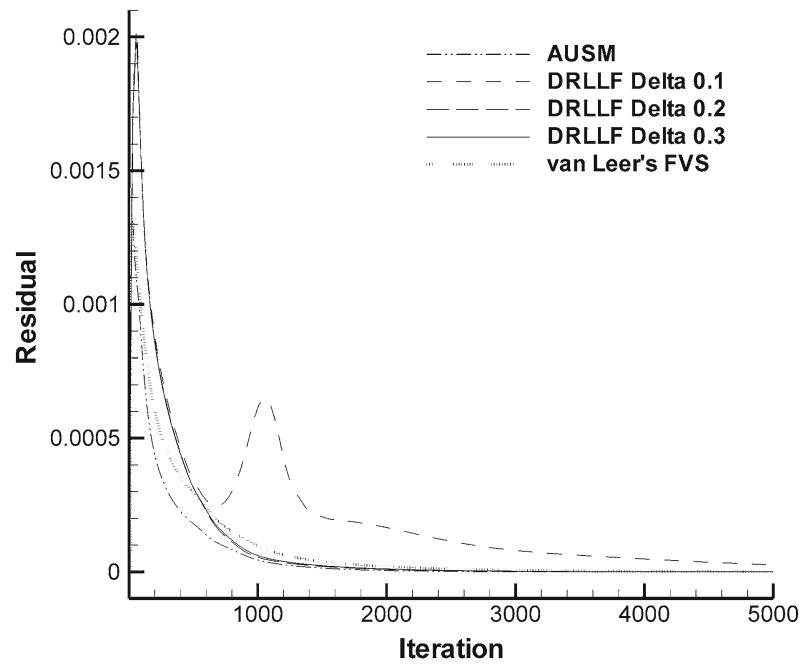

Fig. 12 Residual history plots for the isothermal wall condition. Gradients at the cell-interfaces are computed using central-differencing

trend of the entropy profile is expected to be similar to the temperature profile. Figures 10 and 11 show the increase in specific entropy profile at the trailing edge of the plate for isothermal and adiabatic wall conditions under steady state.

It appears from Figs. 6, 7, 8, 9, 10 and 11 that for the DRLLF scheme, $\delta=0.1$ gives the results closest to the AUSM scheme. However at such low value of $\delta$ the residual diminishes in an oscillatory fashion. This is evident from Figs. 12 and 13, which show the $L_{2}$-norm 


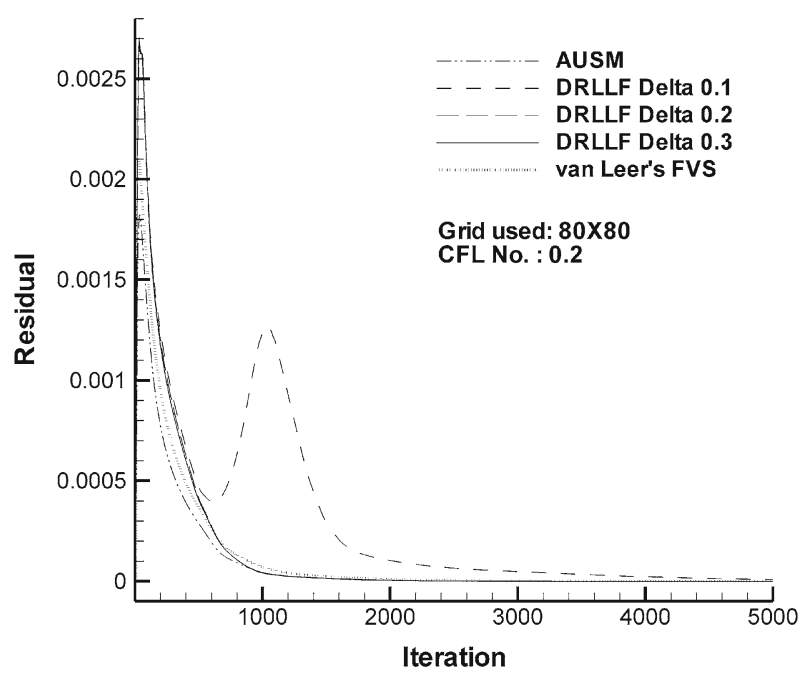

Fig. 13 Residual history plots for the adiabatic wall condition. Gradients at the cell-interfaces are computed using central-differencing

residual history plots for the isothermal and adiabatic wall conditions computed using the different schemes. However at higher values of $\delta$, the convergence of the DRLLF scheme is found similar to van Leer's FVS and AUSM schemes. Thus based on a trade-off between accuracy and smooth convergence rate, $\delta=0.2$ is the most suitable value for evaluating the DR parameter in the computation of this class of problems.

\section{Gradient Computation at Cell-Interfaces Using Green's Theorem}

The $u$-velocity profile at the trailing edge for the isothermal and adiabatic wall conditions using the different inviscid flux schemes are shown in Figs. 14 and 15, where the gradients in the viscous fluxes are computed by using Green's theorem. The very high boundary layer thickness computed by the van Leer's FVS scheme affirms that the scheme is not suitable for viscous flow computations. The velocity profiles obtained by using central-differencing and Green's theorem as shown in Figs. 6, 7, 14 and 15 respectively are similar. The temperature and entropy profiles at the trailing edge as well as the residual history plots for the Green's theorem are also found similar to those for the central-differencing method, which demonstrate the equivalence of the two gradient computation methods on a regular Cartesian grid.

\section{Comparison of CPU-Time}

The same machine and compiler are used for the comparison of the computational time for the different scheme combinations. The processor is Intel(R) Core (TM) 2 Duo T5550 with a processor speed of $1.83 \mathrm{GHz}$. and having 1GB RAM. The Microsoft Visual C++ 6 compiler is used to compile all the codes. Table 1 lists the CPU time for 5000 iterations by the different scheme combinations for the isothermal wall condition. A similar trend is observed for the adiabatic wall condition also.

From the residual history plots, it is seen that AUSM, DRLLF $(\delta=0.2)$ and van Leer's FVS schemes converge almost after the same number of time-steps. However, van Leer's FVS is found to give poor accuracy for viscous flow computations. For the present computations, 


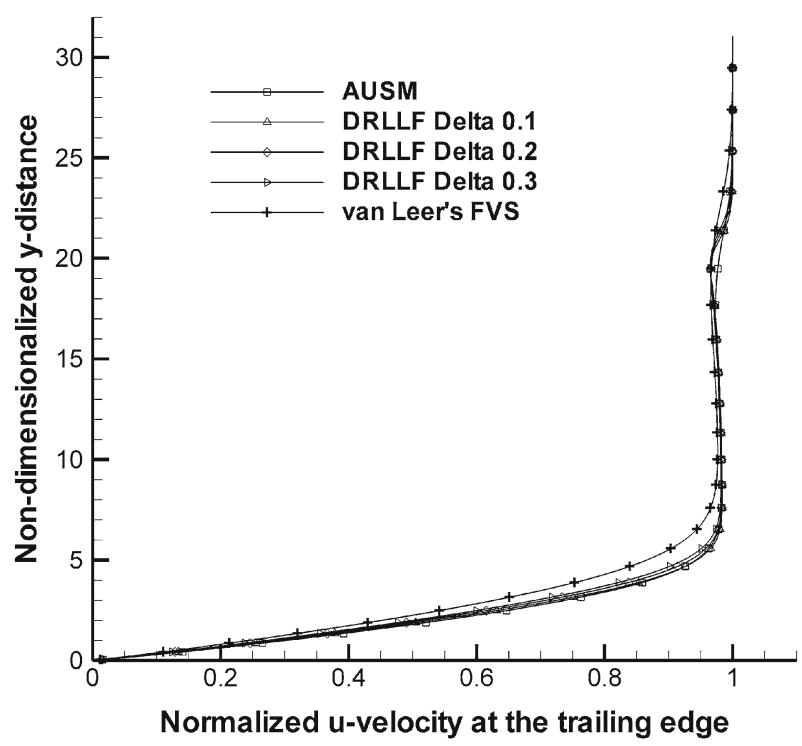

Fig. 14 Trailing edge $u$-velocity profile for the isothermal wall condition. Gradients at the cell-interfaces are computed using Green's theorem

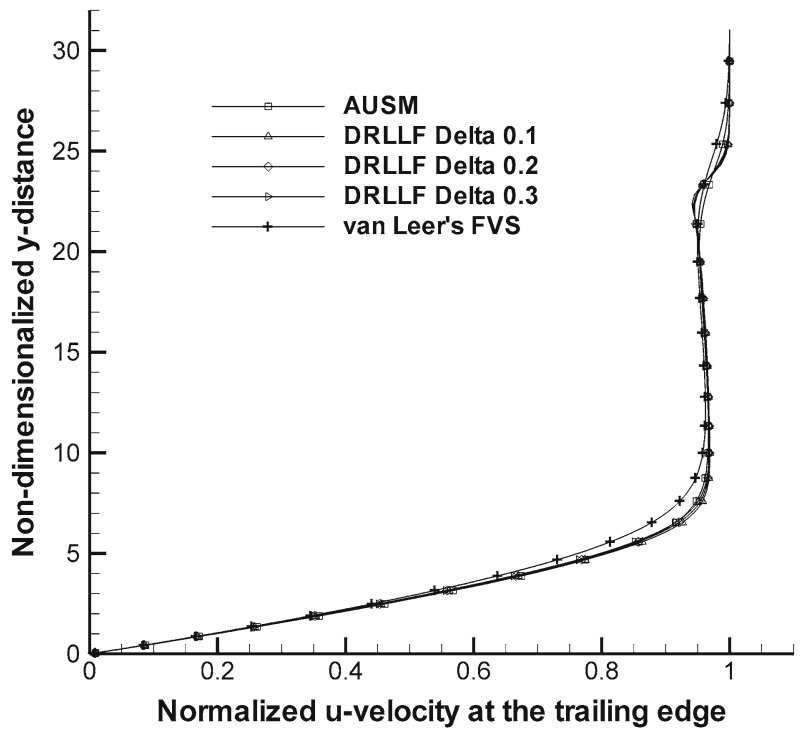

Fig. 15 Trailing edge $u$-velocity profile for the adiabatic wall condition. Gradients at the cell-interfaces are computed using Green's theorem

the DRLLF $(\delta=0.2)$ scheme is found with higher overall accuracy than the AUSM scheme. In addition table 1 reveals that DRLLF scheme consumes marginally less CPU time per iteration than AUSM scheme. This can be attributed to the algorithmic simplicity of the former scheme. For large viscous computations, the saving in computational time by using DRLLF scheme may be significant compared with AUSM scheme, without significant compromise in accuracy. 
Table 1 Comparison of CPU time by various scheme combinations for 5000 iterations

\begin{tabular}{lll}
\hline Inviscid flux scheme & Gradient computation scheme & CPU time (s) \\
\hline AUSM & Central-differencing & 145.61 \\
& Green's theorem & 160.08 \\
DRLLF $(\delta=0.2)$ & Central-differencing & 141.39 \\
& Green's theorem & 155.987 \\
van Leer's FVS & Central-differencing & 139.183 \\
& Green's theorem & 150.476 \\
\hline
\end{tabular}

\section{Summary and Conclusions}

The behaviour of the DRLLF scheme with respect to the computation of high-speed viscous flows is an unexamined area. In the context of computation of viscous supersonic flow past a flat plate, a study is carried out here to examine whether it is possible to use lower values of numerical diffusion parameter in the DRLLF scheme, than stability permits in the computation of inviscid flows. This study shows that reduced artificial viscosity is indeed a possibility. Considering both accuracy and smooth convergence a lower value of the numerical diffusion parameter $(\delta=0.2)$ is suggested for computing such flows. However, a comprehensive mathematical formulation for the regulation of the numerical diffusion applicable to a wide range of compressible flow conditions can further be framed. The overall accuracy of the computation using a smaller DR parameter is high because it resolves the boundary layer as accurately as the AUSM scheme whereas it resolves the shock better than the other two schemes, viz. AUSM and van Leer's FVS. The DRLLF scheme is also comparable with the AUSM and van Leer's FVS schemes in terms of CPU time per iteration. Limitation of the van Leer's FVS scheme for viscous flow computations due to high numerical diffusion is affirmed again. For isothermal wall condition, the location of the point of maximum temperature shifts upward when the numerical diffusion increases and the trends of the temperature profiles above and below this point reverse for different numerical schemes. For adiabatic wall condition, a more diffusive scheme predicts a higher wall temperature. On a regular orthogonal grid, the Green's theorem is comparable with the central-differencing method for the computation of the gradients across cell-interfaces.

\section{References}

1. Abbas, Q., Nordström, J.: Weak versus strong no-slip boundary conditions for the Navier-Stokes equations. Eng. Appl. Comput. Fluid Mech. 4, 29-38 (2010)

2. Aliabadi, S.K., Ray, S.E., Tezduyar, T.E.: SUPG finite element computation of viscous compressible flows based on the conservation and entropy variables formulations. Comput. Mech. 11, 300-312 (1993)

3. Anderson, W.K., Thomas, J.L., van Leer, B.: A Comparison of finite volume flux vector splittings for the Euler equations. AIAA J. 24, 1453-1460 (1986)

4. Aoki, K., Kanba, K., Takata, S.: Numerical analysis of supersonic rarefied flow gas flow past a flat plate. Phys. Fluid (1994-present) 9, 1144-1161 (1997)

5. Arnal, D., Vermeerch, O.: Compressibility effects on laminar-turbulent boundary layer transition. Int. J. Eng. Syst. Model. Simul. 3, 26-35 (2011)

6. Blazek, J.: Computational Fluid Dynamics; Principles and Applications, vol. 1. Elsevier, Amsterdam (2001) 
7. Cebeci, T., Shao, J.P., Kafyeke, F., Laurendeau, E.: Computational Fluid Dynamics for Engineers. Springer, Berlin (2005)

8. Delanaye, M.: Polynomial reconstruction finite volume schemes for compressible Euler and NavierStokes equations on unstructured adaptive grids. Ph.D. Thesis, University De Liege, pp. 15-19 (1996)

9. Deshpande, V., Eshpuniani, B., Sanghi, S.: Computation of supersonic flow over a flat plate with moving protrusion. In: Proceedings of the 37th National and 4th International Conference on Fluid Mechanics and Fluid Power (FMFP10-HS-01), IIT Madras, Chennai, India (2010)

10. Drikakis, D., Durst, F.: A numerical study of viscous supersonic flow past a flat plate at large angles of incidence. Phys. Fluid (1994-present) 6, 1553-1573 (1994)

11. Dwight, R.P.: Heuristic a posteriori estimation of error due to dissipation in finite volume schemes and application to mesh adaptation. J. Comput. Phys. 22, 2845-2863 (2008)

12. Eckert, E.R.G.: Survey of heat transfer at high speeds. WADC Technical Report, pp. 54-70 (1954)

13. Fang, J., Li, Z., Lu, L.: An optimized low-dissipation monotonicity-preserving scheme for numerical simulations of high-speed turbulent flow. J. Sci. Comput. 56, 67-95 (2013)

14. Iyer, P. S., Muppidi, S., Mahesh, K.: Boundary layer transition in high-speed flows due to roughness. AIAA Paper 2012-1106 (2012)

15. Jaisankar, S., Raghurama Rao, S.V.: Diffusion regulation for Euler solvers. J. Comput. Phys. 221, 577-599 (2007)

16. Jaisankar, S., Sheshadri, T.S.: Directional diffusion regulator (DDR) for some numerical solvers of hyperbolic conservation laws. J. Comput. Phys. 233, 83-99 (2013)

17. Jameson, A.: Analysis and design of numerical schemes for gas dynamics 1: artificial diffusion, upwind biasing, limiters and their effect on accuracy and multigrid convergence. Int. J. Comput. Fluid Dyn. 4, 171-218 (1995)

18. Jayantha, P.A., Turner, I.W.: A comparison of gradient approximations for use in finite volume models for two-dimensional diffusion equations. Numer. Heat Transf. Part B 40, 367-390 (2001)

19. Kalita, P., Dass, A.K.: Computation of high speed flows using diffusion regulation. In: Proceedings of the 40th National Conference on Fluid Mechanics and Fluid Power, NIT Hamirpur, pp. 1231-1239 (2013)

20. Kannan, R.: A high order spectral volume formulation for solving equations containing higher spatial derivative terms: formulation and analysis for third derivative spatial terms using the LDG discretization procedure. Commun. Comput. Phys. 10, 1257-1279 (2011)

21. Kannan, R.: A high order spectral volume formulation for solving equations containing higher spatial derivative terms II: improving the third derivative spatial discretization using the LDG2 method. Commun. Comput. Phys. 12, 767-788 (2012)

22. Kannan, R., Wang, Z.: Improving the high order spectral volume formulation using a diffusion regulator. Commun. Comput. Phys. 12, 247-260 (2012)

23. LeVeque, R.J.: Finite Volume Methods for Hyperbolic Problems Cambridge Texts in Applied Mathematics, 1st edn, pp. 232-233. Cambridge University Press, Cambridge (2002)

24. Liou, M.S., Steffen Jr, C.J.: A new flux splitting scheme. J. Comput. Phys. 107, 23-29 (1993)

25. MacCormack, R.W.: The effect of viscosity in hypervelocity impact cratering. AIAA Paper, Cincinnati, Ohio, 69-354 (1969)

26. Puppo, G.: Numerical entropy production on shocks and smooth transitions. J. Sci. Comput. 17, 263-271 (2002)

27. Roe, P.L.: Approximate Riemann solvers, parameter vectors and difference schemes. J. Comput. Phys. 43, 357-372 (1981)

28. Savelyev, A.D.: Numerical simulation of laminar viscous supersonic flow past a two-dimensional cavity. Fluid Dyn. 29, 759-764 (1994)

29. Steger, J.L., Warming, R.F.: Flux vector splitting of the inviscid gasdynamic equations with application to finite-difference methods. J. Comput. Phys. 40, 263-293 (1981)

30. Tu, J., Yeoh, G.H., Liu, C.: Computational Fluid Dynamics: A Practical Approach, 1st edn. ButterworthHeinemann, London (2008)

31. Van Driest, E.R.: Investigation of laminar boundary layer in compressible fluids using the Crocco method. NACA Technical Note 2597 (1952)

32. Xu, L., Weng, P.: High order accurate and low dissipation method for unsteady compressible viscous flow computation on helicopter rotor in forward flight. J. Comput. Phys. 258, 470-488 (2014) 